\title{
The emergent qualities of digital specimen images in biology
}

\author{
Christine Hine
}

Department of Sociology

University of Surrey

Guildford, Surrey, GU2 7XH, UK

c.hine@surrey.ac.uk

Author's draft. Published as Hine, C.2012. The emergent qualities of digital specimen images in biology. Information, Communication and Society._Published online before print June 25, 2012, doi: 10.1080/1369118X.2012.696124

\begin{abstract}
Whilst digital technologies are often popularly portrayed as inherently different from their material counterparts, recent research has accentuated continuities between the two. Research on the material aspects of digital technologies has emphasised that both material and digital technologies are embedded in practice and acquire their meaning in context. This is particularly so in science, where research in science and technology studies has illuminated the contextual interpretation of representations and their contingent manifestation through embedding in specific sociotechnical configurations. The current paper explores how digital technologies are experienced in a specific field of science, biological systematics. Email accounts were solicited from biologists who have been working with digital images of the biological specimens conventionally used in work on the classification and naming of organisms. Thematic analysis of the interviews shows that qualities of digital images were highly contextual, often defined in dialogue with their material counterparts which are also defined in fluid and contextual fashion. Discussing the use of digital specimen images
\end{abstract}


involved distinctions between different forms of work and different organisms being studied and referenced the varied institutional and geographic positioning of respondents. The introduction of digital images offered the possibility of new sociotechnical configurations emerging and to some extent realised the aspirations of digitization projects to enable new forms of distributed working. This was, however, a qualified success restricted to only some aspects of the systematists' work.

\section{Introduction}

Early in the mainstreaming of the Internet it become almost a taken-for-granted assumption that digitization of collections of precious objects was a good thing (Smith 1999), with the 1990s described as a “decade of digitization” (Lee 2001). Digitization projects to make copies of collections of art works, historical archives, books and biological specimens have continued to proliferate in recent years. Rendering precious and rare objects in digital format is seen as making them more widely available, and thus democratising access whilst preserving the original physical artefact undiminished. Given widespread belief in the virtues of the digital, a "computerization movement” (Kling and Iacono 1996; Iacono and Kling 2001; Hine 2006) often takes over, such that detailed justifications in terms of potential use are rarely required for each digitization project and the precise form of "access” which digital images are to promote is rarely specified (Ester 1994; Terras 2008). Actual uses are thus often discovered after the event, as practitioners working in the field explore the qualities of the newly available digital collections and find ways to fit them into their working practices.

As practitioners become conscious that practices are beginning to change they may be particularly willing to reflect upon the nature of the change they are experiencing, before a complete “black-boxing” (Latour 1987) of the new way of doing things has occurred. This 
paper focuses on a group of professionals at the point where they are beginning to make routine use of digitized artefacts, and explores their perceptions of the objects and practices concerned. In particular, this offers the opportunity to look in detail at the extent to which the digital artefact is seen as substituting for its physical counterpart, often a key claim in the rationale for digitization projects. In detailing how the digital artefacts are appropriately used practitioners indulge in reflexive moments which involve making fine distinctions between different forms of work, different professional identities and geographic locations and different kinds of physical and digital artefact.

This paper focuses on a domain of professional practice in which digital artefacts have increasingly come to be used alongside and instead of their physical counterparts. The domain is the branch of biology concerned with the classification and naming of organisms, known as taxonomy, or systematics. Professionals working in this field have traditionally used collections of preserved specimens to represent the diversity of living organisms. Recently many specimen collections have become available via the Internet in digital format, initially via online catalogues and latterly as collections of digital images. Taking as its backdrop the computerization movements which promoted the production of digital specimen image collections (Hine 2006; Hine 2008), this paper analyses accounts collected via email from taxonomists working with digital images in 2005/6, at a point when digital collections of specimens had become widely available but their use not yet entirely commonplace. The paper first briefly introduces the recent digitization movement in systematics, and then outlines perspectives from research in new media and representation in scientific practice to justify an expectation that the qualities of digital and physical objects will be emergent in contexts of practice. A description of the methodology for collecting accounts from practitioners then sets the scene for presentation of the findings. A concluding section 
evaluates the potential for wholesale shift towards forms of digitally enabled distributed working in systematics.

\section{Systematics and digitization}

Biologists working in systematics are often based not in university science departments but in natural history museums, herbaria or botanic gardens which may or may not be affiliated with universities. Traditionally systematists have examined collections of specimens (which might for example be animal bodies preserved in alcohol, dried plants pressed upon a page, a fossil fragment of an extinct organism or a microbial culture contained in a phial). These preserved artefacts are used as enduring reference points for identification and as material for exploring relationships between organisms, acting in effect as a "library of nature”. Routinized practices for preserving, organizing, owning, sharing and examining these specimens are in place. The outcomes of work in systematics are published as scientific papers or as a variety of different, conventionalised forms of publication including checklists, inventories, diagnostic tools or authoritative treatments of a group or species and, increasingly, as elements of a publicly available database. In addition to needing access to the literature, in order to do credible work on the classification of a group of organisms a systematist will need access to an appropriate set of specimens to examine. An established system of loans and visits allows systematists to assemble a set of specimens for the task at hand from the various collections in which they might be held, so that they can compare characteristics and identify diagnostic features.

Latterly there has been a growth in use of digital images of specimens to supplement or replace examination of the material specimen itself. As Hine (2008) describes, a variety of factors have driven the expansion of online collections of digital specimen images which are 
provided, in large part, by the institutions that hold the material specimens. One set of factors promoting growth in digital specimen image collections revolves around concerns about the inefficiency of traditional working practices, in the face of the massive task facing the profession of cataloguing as yet undescribed species. This is an issue of international concern in the face of global recognition of threats to biodiversity, and major institutions have found it necessary to invest in digital resources as a response to national and international pressures to share expertise. It has also become politically expedient, for the sake of the reputation of the discipline and to ensure its continued fundability, to be seen to innovate and invest in digital technologies. Another motivating factor for developments in digital specimens builds on concerns that collections are largely located for historical reasons within developed nations rather than the biodiversity hotspots from which the specimens originated. Digital specimens have been promoted as part of a perceived moral duty to widen access to specimen collections and overcome inequalities between scientists from different countries.

At the time when the accounts analysed in this paper were generated (2005/6), there was a considerable momentum around the production of digital images of specimen collections and the skills to achieve this were becoming more widespread (Häuser et al. 2005). Digitization of specimens remains a contemporary concern but problems in achieving large scale digitization also persist: for example, discussion of ways to organize and speed up digitization of existing collections remained a popular topic for papers presented at the 2011 meeting of the Taxonomic Databases Working Group ${ }^{1}$ and an ongoing NSF funding programme in the United States focuses on Advancing Digitization of Biological Collections. Recent publications continue to discuss the need to speed up digitization to make collections

\footnotetext{
${ }^{1}$ The TDWG (http://www.tdwg.org/) is the key body which formulates standards for biodiversity informatics projects, with the goal of ensuring maximum interoperability of data from diverse projects.
} 
more accessible, and highlight the challenges of prioritization (Scoble 2010; Vollmar et al. 2010). As Hine (2008) describes, a complex politics and considerable practical constraints influence what is made available, by whom and in what format. However, although there is considerable pressure to digitize collections, it is still often not entirely clear who actually uses the resulting digital specimens, nor what they feel that they can usefully achieve with them. Little is known about how digital specimens are being embedded in practice, or to what extent examining a digital specimen is viewed as an acceptable way to understand an organism. Against this backdrop the current paper describes the emergent meanings of digital specimens in the accounts of working systematists.

\section{Digital practices and representation in science}

Once the initial surge of hype about the transformative properties of the Internet and other digital media had passed, interest increasingly turned to exploring how these technologies become embedded into everyday life, and a focus on "micro-scale meanings and experiences of new media use” (Lievrouw 2004: 13) became a predominant theme in new media research. Digital technologies do not float free of other forms of lived experience, but are embedded in and experienced through various existing social, spatial and temporal structures, acquiring diverse meanings along the way. New media have undergone what Graham (2004) describes as a banalization, as they sink into the fabric of everyday life and become a routine part of daily experience. With this in mind, the current paper focuses on a moment when new practices were just becoming embedded, asking practitioners to reflect on the emergent routinization of work with digital artefacts before it became wholly taken-for-granted. It is also significant that this paper does not focus on a leisure context, within which there might be a certain freedom to develop innovative uses of digital media as a part of an expression of distinction or statement of social identity. Instead it focuses on professionals using digital 
images in their work. Introduction of a new form of representation can offer a significant reflexive moment when professional working practices and values are closely examined and existing relationships re-evaluated (Boland et al. 2007). In many fields of professional practice particular objects are held to be very important in successful accomplishment of work, and new representations can be controversial.

Science offers a particularly potent example of a field concerned with the nature of representation. Within scientific practice technologies of representation play an important role in rendering scientific phenomena amenable to study. As Burri and Dumit (2008) describe in a comprehensive review, the production, consumption and dissemination of scientific images has been the subject of many studies from diverse theoretical perspectives. Prominent among the insights from the sociology of science has been the notion that the adequacy of particular representations is contextually negotiable and that representations acquire meaning within complexes of activities and within particular technological, methodological and institutional contexts (for example Lynch and Woolgar 1990; Beaulieu 2002; Joyce 2005). Images must participate in a "visual culture” (de Rijcke and Beaulieu 2007) to be treated as effective representations. It stands to reason then that a new way of producing representations, such as offered by digital media, will need to be embedded within a set of practices within which it makes sense. These practices may be new, but they will need to be presented as adequate to the task at hand, to an audience of peers. Choices of particular imaging technologies can also carry consequences for identity and “doing distinction” among professionals (Burri 2008). As Coopmans (2011) argues, images not only represent but are also represented, in particular circumstances for particular audiences. It is important to keep in mind, therefore, that interviews with practicing scientists about their use of digital images will provide accounts of practice in an emerging visual culture, but that 
these accounts will be presented in a context in which the professional is concerned to portray this practice as the action of a responsible professional behaving in line with the expectations of the field.

Research undertaken from a science and technology studies perspective argues that the assumed qualities of digital objects are not necessarily inherent in them simply because they are digital. Even the widely accepted mobility of digital images takes work to achieve, as demonstrated in Coopmans’ (2006) ethnographic study of the development of a database of digital mammogram images. Coopmans proposes that a sociological approach to data such as these digital images involves looking carefully at what it means, and what effort it takes, for data to be experienced as mobile. Coopmans also cites Mort et al's (2003) study of telemedicine to demonstrate the additional forms of information and labour that practitioners draw upon in order to embed images into meaningful medical practice. In this context practitioners do not experience the image, on its own, as an adequate representation of the patient. Coopmans suggests that we need to develop "an understanding of the process of digitizing, not so much as the translation of medical images into a new (binary) form, but as their incorporation into a new sociotechnical configuration” (Coopmans 2006: 7). Bearing in mind the caveats above about the production of accounts being shaped by the concern to portray oneself as a responsible professional, it then becomes significant to ask, in the case of digital specimen images, under what circumstances the digital image is considered an adequate representation for taxonomic purposes. What supporting infrastructure is needed in order to use digital specimen images and how does the image relate to the physical specimen collection, and the living organism? 
Representations in science are inherently selective, showing particular aspects of the phenomenon being studied, for specific purposes. They emerge, as Burri and Dumit describe it, "from a lengthy series of technological opportunities and constraints, negotiations and decisions” (2008: 301). The ability of a particular form of image to persuasively and economically portray information relevant to what the scientist perceives as the task at hand is key (Burri 2012). The most detailed image is not necessarily considered the best. For example, in systematics a line drawing has sometimes been considered a superior form of representation to a photograph, since details considered “distracting noise” (Bell and Bryan 2008: 22) can be deliberately excluded from the line drawing. It is therefore important to remember that there will be many different possible ways of rendering a specimen in the form of a digital image, and that some will be found more useful than others for particular kinds of scientific work. The digital technology is not expected to be positioned as being universally inadequate or adequate in itself in relation to a "real” object with fixed qualities (particularly so in this case because the physical specimen itself is a preserved artefact which only very selectively portrays the qualities of the living organism). Rather, the qualities of digital and material artefact are likely to be assessed in relation to one another, in context of particular sets of activities and priorities.

This paper looks at accounts offered by scientists using digital images alongside and as substitutes for the material artefacts that have hitherto been the focus of their practice. Whilst there is a broad agreement about many of the professional practices and implicit values within systematics, there is also considerable diversity. Practitioners have considerable ability to choose the organization of their work, the form of outputs and the technologies which might be used to achieve them. They enjoy a large degree of autonomy in carrying out their work, and yet the results will ultimately be judged by their peers. They also work on very 
different organisms, which themselves require quite specific ways of working. This therefore offers an interesting location in which to study the emergence of diverse digital practices and situated accounts of adequacy. In order to explore the diversity of perceptions of digital images I sought accounts from a range of practitioners working on different taxonomic groups, in different geographic locations and different institutions. The following section describes how these were generated.

\section{Methodology}

Participants to the study were recruited from Taxacom, a mailing list for biological systematics in existence since 1987 with a current subscribership of around 2,000. The list is used by a diverse array of professionals with an interest in systematics, including both those working in the major systematic institutions and those only peripherally concerned with systematics as one aspect of their work. Discussions cover a wide range of theoretical and substantive issues, alongside job advertisements and practical requests for help in obtaining specimens and literature. Many participants monitor list discussions in order to keep abreast of developments in the discipline. I hoped, by appealing for research participants via the Taxacom list, to encourage participation from people working in a wider range of situations than I would achieve via face-to-face interviews. I made a request specifically for research participants who were making use of online specimen images in their work. My email asked them to describe their practices with digital images, as follows:

I'd be really grateful to hear from anyone who has made use of online specimen images in their research work, and would be willing to spend a little time reflecting on how useful they were, and how they might have added to or changed your previous ways of working. Can you look at a broader range of material than before by using 
online images, or do you still tend to end up requesting loans and making visits? How do you manage side by side comparisons between digital images and real specimens? Has your working environment changed: do you work within the collection, or in front of the computer - or does your working environment combine the two? What is the experience of collection managers - has putting images online increased or decreased demand on the original collection? I'm interested in general reflections but I'd also like to hear about any particular instances where an online image was either really helpful, or failed to do the task asked of it.

After an initial wave of responses tailed off I sent a follow-up message to the list to encourage further responses. Those who replied to either of these initial emails were sent tailored responses with additional questions which asked them to further explain or contextualise their answers. 22 people in total responded (see Table 1). Of these, eight responses were only a few lines long (although even these contained useful data), but the majority extended to several paragraphs of text describing practices and projects in detail or became longer conversational exchanges in which the participant and I explored aspects of their work with digital specimen images in depth. Several respondents gave links to websites or published works. These links were followed and articles retrieved to provide context for interpreting the accounts, and in some cases examining these led me to additional questions. Respondents who were developers of online image resources as well as users were asked follow-up questions about how their resource came to be developed and what they knew about their users. The resulting accounts were subjected to a thematic analysis which focused on the qualities ascribed to digital and material specimens by the respondents, the different ways in which they saw them as objects to work with and the distinctions made in terms of professional identities and forms of work. 
As compared to face-to-face interviews the accounts which were generated by this process were shorter, but dense in relevant information. Some of the accounts were clearly written over a period of time as a result of serious reflection. The asynchronous approach to research participation offered by email can be an advantage not only where a population is geographically dispersed, but also where we hope to generate respondent accounts based on extended reflection (Illingworth 2001; McCoyd and Kerson 2006). We might expect the account offered in an email to be different to that which might be given face-to-face, but neither is necessarily to be taken as a distortion of the other (Orgad 2005). An additional advantage of email exchanges for the purpose of this study was the readiness with which participants could include additional information. They were not just confined to the spoken word as in a face-to-face or telephone interview, but were able to offer multimedia accounts combining first person text with links to websites and published papers to illustrate their points and direct me to resources they thought relevant. Email-generated accounts therefore appeared to be an appropriate tool for generating relevant data from this population, although this technique is obviously not without limitations and would not be appropriate if spontaneous accounts or direct observations in which the role of digital artefacts was not deliberately topicalised were required

\section{The qualities of digital and material specimens as objects of systematics}

Embedded in recognisable practice

Participants did not, on the whole, present digital specimen images as radically transformative of their practice. Some said that viewing specimens online might make a particular project feasible to achieve in the short term, rather than waiting until loan specimens arrived or there was sufficient time and funding to travel, but nobody said that 
their work had dramatically changed in direction or scope. Instead, they stressed continuity of both practices and fundamental values, portraying digital specimen images as fitting into their existing work, albeit sometimes making it faster or more efficient. The online availability of images was often described using familiar metaphors. One respondent described an online image search as "a lot like going into the field - you never know what you will find!” (Respondent 2), while another described his own web site as "my personal digital encylopedia” (Respondent 15). In one case two familiar metaphors were used to distinguish the type of work for which digital images were useful: "so I use the images like a huge field guide, but NOT like a huge museum collection.” (Respondent 18).

Respondents positioned digital images as better for some kinds of work than others. In particular, just as with the respondent who described digital images as field guide rather than museum collection, identification work was often distinguished from taxonomic work involving revisions to classifications. Enthusiasm about digital specimens was much greater in context of identification, and considerable caution expressed by many about the possibility of doing detailed taxonomic work on the digital artefact. Respondents were careful to portray themselves as responsible members of their profession, making judgments which were based on adequate examination of evidence. Their portrayals of the contextual adequacy of digital specimens sometimes referenced explicitly what a responsible member of the profession would be expected to do. For example, one respondent problematised the digital image because it did not participate in the prevailing convention that examining a material specimen conferred a responsibility to correct any misinformation: “one important advantage of a specimen in hand (v. digital) is that if it is misidentified, then as the specialist you are obliged to correct the identification with an annotation slip" (Respondent 5). This distinction suggests a certain distancing from the digital specimen image, such that one is not completely in its 
presence (although it should be noted that many digital taxonomic resources do attempt to encourage experts to correct erroneous entries within their area of expertise (see, for example, International Plant Names Index 2004)).

A further distinction which referenced the adequate performance of the systematist's role was the examination of type specimens. The type specimen has a very tightly demarcated status in systematics. A type is nominated when the species is first described, and remains the key reference point for determining whether the name of the species is being applied correctly. Apart from a few very circumscribed circumstances where an image can substitute, the type specimen must be a physical instanciation of the organism in question. It is expected that anyone working on the revised classification of a group will have examined all of the type specimens, and yet institutions are very reluctant to lend out type specimens due to their unique, irreplaceable status. Digital images of type specimens have consequently become very useful. As one respondent described it: "I do make concerted efforts to find type specimens online and in those cases (nearly always) a relatively quick viewing of the specimen (20 seconds or less) is typically enough to confirm whether or not it is a type and whether the specimen matches the name that is (at least generally by most authors) being used for that species in the literature” (Respondent 5). In publications describing a revised classification it is routine for authors to note which specimens they have examined, and in this instance the respondent explained that he had added a note in one of his publications that a type had been "seen digitally”, expressing his judgment that examining a digital type specimen counted as an acceptable practice, but still a notable one.

It was clear from the descriptions respondents gave that embedding digital specimens in practice involved more than simply working out, in a functional sense, what a digital 
specimen could do. Judgments about the capacities of digital specimens were embedded in the formal practices of the discipline, such as the definition of type specimens, but also in moral judgments about how a responsible systematist would act. In many circumstances an act of examining a digital image as opposed to a material specimen was still viewed as remarkable, and something which one should responsibly demarcate as not having been quite the real thing.

Speed, searchability, manipulabilty

Amongst the topics of reflection which discussing digital specimens occasioned, the analysis of how one worked with specimens and why one did so was prominent. In this context the predominant quality attributed to digital specimens is their fast availability, as compared to a loan specimen for which one might have to wait some time. Even where the material specimen was in a local collection, the digital specimen might still have an advantage in accessibility, as this description from a fish taxonomist outlines:

And it takes a lot less time to call up the images on my computer screen, than it does to get up from my desk, walk into the collection, find the actual specimen, pull it off the shelf (sometimes requiring a ladder), and take it out of the jar (not to mention the need to return it properly to the jar and the shelf, and the potential damage to the specimen itself), etc., etc. (Respondent 8).

Although digital specimens were often praised because one could get hold of them fast, speed was not universally valued. Identifying an unknown organism via comparison with a digital specimen was generally portrayed as quick, but also qualified as provisional, requiring confirmation from an expert, or risky. The developer of one resource including specimen 
images described it as a "front-line or first port-of-call resource" and stressed that certain identification would need the involvement of an expert (Respondent 1). One respondent went to some lengths to explain to me how he worked with material specimens, formulating a portrayal of his practice as an almost meditative form of co-presence with the object:

Well, in the early stages of doing taxonomic work on poorly known groups -- what I do a lot of, since many specimens represent undescribed (new to science) species -- it often helps to spend some "slow time" looking at specimens, one by one, and just allowing your mind to shut off the internal dialogue apart from asking and answering short questions about the specimen in view. (Respondent 5).

In this context digital specimens, for him, lacked the necessary qualities to achieve a slow copresence.

Sometimes the digital image was valued not for its portrayal of authentic qualities of the material specimen itself, but more for the fact that it confirmed the existence and location of the material specimen, or allowed details of the label rather than of the specimen itself to be examined. Material specimens were characterised as troublesome to locate, and by comparison the digital specimen became a particularly valuable resource in so far as it was positioned within a searchable territory, either in a discrete database or via a Google images search. In material specimen collections there is often no full database or catalogue, and a specimen will be locatable only in so far as the physical arrangement of cabinets reflects the accepted taxonomic groupings. In this context, and for certain kinds of work, the searchability and locatability of the digital specimen images become their most notable characteristic. 
Beyond being locatable, specimens are valued as objects to work with. The "slow time" with specimens that detailed taxonomic work involves often entails manipulating and actively examining specimens to reveal key characteristics. Sometimes this can be achieved with digital specimens: respondents talked of being able to zoom in and explore the image, of being able to compare and juxtapose, and of working, in some cases, with material and digital specimens side by side. However, in many cases digital specimen images were not seen as appropriately manipulable: one respondent described how varying the precise angle at which light fell on a material specimen enabled the patterns of oil glands on a leaf surface to be discerned (Respondent 5). Others doubted the ability of a digital image ever to match the qualities of the material specimen: "I work with grasses and the parts overlap each other in such a way that it is rare that even a high res image will show what I need” (Respondent 12). For any specified group of organisms a different kind of image, focusing in on a different structure might be required in order to be seen as making it useful for taxonomic work. Again, community standards were referenced as much as one’s own judgment in deciding whether a particular kind of work could be done with a digital specimen. As one respondent put it:

\footnotetext{
I have collected a great number of measurements from physical specimens that I have analyzed using PCA, but I have not and almost certainly would not collect such data from specimen images, as I would have a difficult time convincing myself \& others of the two types of data are similar enough to compare rigorously. (Respondent 7)
}

The physical specimen often operates as a gold standard against which the digital specimen image falls short, particularly when an active form of co-presence with the specimen is 
required. There were, however, instances where the relationship between specimen, image and organism was more complex, as explored below.

\section{Specimen/image/organism relationship}

Instances in which a digital image might be superior to a physical specimen were mentioned by some respondents, including the same respondent who described the inability to take measurements reliably from a specimen image:

However, given the nature of nematode specimens a good sequence of through-focus images may be of great use, both as it is easily transmitted and may actually retain more fidelity than the degraded original specimen. Regardless of preparation care, many nematode specimens degrade over a few decades so permanence of types is uncertain. (Respondent 7).

Similarly, an image of a fresh fish specimen was said to allow access to the life-colour in the way that an older preserved specimen could not. There was some evidence that availability of digital images of living organisms, or images of fresh specimens, might challenge the traditional status of the preserved specimen as the primary focus of taxonomic work:

My other frustration with the approach is that living plants are far more attractive than dead plants and that a series of pictures taken of a living plant, that is then killed and made into a voucher specimen, is of greater value than 100 thoughtless herbarium specimen images. (Respondent 12) 
As described earlier, discussing the contextual adequacy of a digital specimen image for a particular kind of work also entails defining what that work is and what can be done with physical specimens. On the whole, the practice of working with digital specimen images that respondents described was still bedded in the sociotechnical configuration (Coopmans 2006) of the specimen collection, with its focus on making comparisons using preserved materials and their accompanying documentation. The suggestions that images of living organisms, or fresh specimens might find uses in taxonomic work not offered by preserved physical specimens offer the intriguing possibility of a different sociotechnical configuration coming into play, retaining the status of the specimen collection as the ultimate repository of voucher specimens, but rendering it less a focus of everyday working practice.

\section{Geographic and economic distinctions}

For some respondents, the usefulness of digital specimens in practice was tied to consciousness of their own position in terms of the prevailing funding climate, constraints on travel, institutional and national positions on the loan of specimens, and international inequalities. A respondent from Uruguay, for example, noted the expansion in the scope of work that he could undertake with the advent of online digital image collections.

I know the pre-Internet epoch, when getting or interchanging information was very difficult in my country, due to a bad post service, expensive international calls, etc. So I appreciate very much the facilities of Internet. [...] In brief, for we inhabitants of $3^{\text {rd }}$ World (euphemism for poor countries) the www has been a very, very useful tool, including online images, being a cheap way to be integrated to the rest of the world. (Respondent 6) 
This discussion of advantages was tempered, however, by the observation that for him nothing could fully replace the physical specimen. Reflection on the constraints on use of physical specimens also came from participants in the United States. One participant described eloquently the challenges of acquiring sufficient specimens both for identification purposes and for taxonomic revisions when one had a limited collection of specimens on site and travel was constrained:

I have used on-line images of type specimens quite a bit over the last 5 years or so. I work at a medium-sized university with relatively poor resources for taxonomic research, and little internal funding for travel. A heavy teaching load virtually precludes any travel outside of the summer to visit other herbaria. (Respondent 5)

For these participants, talking about digital specimens offered up a potent reflexive moment prompting talk about their own place within the discipline. While the numbers here are insufficient for generalization, it appeared that availability of digital specimen images was enabling new kinds of work for at least some otherwise marginalized members of the procession, although they continued to be conscious that the digital resource did not entirely substitute for access to physical specimens.

\section{Conclusion}

Contemporary digitization movements in science participate in a high-level policy interest in promoting e-research and cyberinfrastructure, aimed at encouraging collaboration, increasing the scale of research and enabling distributed working (The Virtual Knowledge Studio 2008; Dutton and Jeffreys 2010). Research into the conditions under which the promised qualities of digital objects materialise in practice could inform discussion of the extent to which 
distributed working in various forms of science is a realisable promise. The number of participants in the study described here precludes generalization, but it does appear that for at least some systematists digital specimen images were enabling a form of distributed working, through seeing specimens at a distance and sharing expertise by consulting distant colleagues on the identification of specimens via images. This work was, however, often carefully distinguished from the forms of work one could do with a physical specimen. Portrayals of the impact of digital specimens were nuanced according to where user and resource were located, by the group of organisms concerned, their physical characteristics and diagnostic features and the state of taxonomic knowledge of the group. Digital specimen images participate in a complex culture of representation in which adequacy is locally negotiated but references a wider expectation of what one should do in order to produce good work.

The digital specimen image was valued for its accessibility and locatability, but often fell short in terms of participant expectations of the kind of manipulation it should allow. Participants frequently did not feel sufficiently co-present as experts with a specimen in its digital form to allow confident representation of themselves as conducting taxonomic revisions. In discussing identification, however, the digital specimen found more favour, and here the "visual value" of being able to take in information at a glance identified by Burri (2012) came to the fore. In the context of identification work, if not in production of taxonomic revisions, the ready availability of digital specimen images might even be said to be promoting visual work practices over other means of identification such as the use of dichotomous keys. In some cases, imaging living organisms, as opposed to specimens offers the possibility of significantly reconfiguring the role of the specimen collection in working practices. It is however certainly not inevitable that digital specimen images or digital images of organisms will prevail as a way of getting work done in systematics, nor indeed that a 
visual logic (Burri 2012) will increasingly dominate. In parallel with the developments described in this paper a movement towards DNA sequence comparison in systematics has emerged, and this offers up a different technology of representation which again redefines the role of the specimen (Ellis 2008) and demands a quite different form of co-presence with the specimen as the appropriate way to do good work.

\section{References}

Beaulieu, A. (2002) 'Images are not the (only) truth: brain mapping, visual knowledge, and iconoclasm', Science Technology \& Human Values, vol. 27, no. 1, pp. 53-86.

Bell, A. D. and A. Bryan (2008) Plant Form: an Illustrated Guide to Flowering Plant Morphology, Portland, Oregon, Timber Press, $2^{\text {nd }}$ edn.

Boland, R. J., K. Lyytinen and Y. Yoo (2007) 'Wakes of innovation in project networks: The case of digital 3-D representations in architecture, engineering, and construction', Organization Science, vol. 18, no. 4, pp. 631-647.

Burri, R. V. (2008) ‘Doing distinctions’, Social Studies of Science, vol. 38, no. 1, pp. 35-62.

Burri, R. V. (2012) 'Visual rationalities: towards a sociology of images’, Current Sociology, vol. 60, no. 1 , pp. 45-60.

Burri, R. V. and J. Dumit (2008) ‘Social studies of scientific imaging and visualization’, in The Handbook of Science and Technology Studies, ed. E. J. Hackett, O. Amsterdamsaka, M. Lynch and J. Wajcman, Cambridge, MA, MIT Press, pp. 297317.

Coopmans, C. (2006) 'Making mammograms mobile: Suggestions for a sociology of data mobility’, Information, Communication \& Society, vol. 9, no. 1, pp. 1-19. 
Coopmans, C. (2011) ““Face value”: New medical imaging software in commercial view’, Social Studies of Science, vol. 41, no. 2, pp. 155-176.

de Rijcke, S. and A. Beaulieu (2007) 'Essay review: taking a good look at why scientific images don't speak for themselves', Theory \& Psychology, vol. 17, no. 5, pp. 733-742.

Dutton, W. H. and P. W. Jeffreys, eds. (2010) World Wide Research: Reshaping the Sciences and Humanities, Cambridge, MA, MIT Press.

Ellis, R. (2008) 'Rethinking the value of biological specimens: laboratories, museums and the Barcoding of Life Initiative', Museum and Society, vol. 6, no. 2, pp. 172-191.

Ester, M. (1994) 'Digital images in the context of visual collections and scholarship', Visual Resources, vol. 10, no. 1, pp. 11-24.

Graham, S. (2004) 'Beyond the 'dazzling light’: from dreams of transcendence to the “remediation” of urban life', New Media and Society, vol. 6, no. 1, pp. 16-25.

Häuser, C. L., A. Steiner, J. Holstein and M. J. Scoble, eds. (2005) Digital Imaging of Biological Type Specimens. A Manual of Best Practice, Stuttgart, European Network for Biodiversity Information (ENBI).

Hine, C. (2006) 'Computerization movements and scientific disciplines: the reflexive potential of e-science', in New Infrastructures for Knowledge Production: Understanding E-Science, ed. C. Hine, Hershey, PA, Information Science Publishing, pp. 26 - 47.

Hine, C. (2008) Systematics as Cyberscience: Computers, Change and Continuity in Science, Cambridge, MA, MIT Press.

Iacono, S. and R. Kling (2001) 'Computerization movements: the rise of the Internet and distant forms of work', in Information Technology and Organizational Transformation: History, Rhetoric and Practice, ed. J. Yates and J. van Maanen, Thousand Oaks, CA, Sage, pp. 93-135. 
Illingworth, N. (2001) 'The Internet matters: exploring the use of the internet as a research tool', Sociological Research Online, vol. 6, no. 2, http://www.socresonline.org.uk/6/2/illingworth.html International Plant Names Index. (2004) How can you help? http://www.uk.ipni.org/how to_help.html (Retrieved 28 July, 2005)

Joyce, K. (2005) ‘Appealing images’, Social Studies of Science, vol. 35, no. 3, pp. 437-462. Kling, R. and S. Iacono (1996) 'Computerization movements and tales of technological utopianism', in Computerization and Controversy: Value Conflicts and Social Choices, ed. R. Kling, San Diego, Academic Press, pp. 85-105.

Latour, B. (1987) Science in Action: How to Follow Engineers and Scientists through Society, Cambridge, MA, Harvard University Press.

Lee, S. D. (2001) Digital Imaging: a Practical Handbook, New York, Neal-Schuman Publishers in association with Library Association Pub.

Lievrouw, L. A. (2004) 'What's changed about new media? Introduction to the fifth anniversary issue of New Media and Society’, New Media and Society, vol. 6, no. 1, pp. 9-15.

Lynch, M. and S. Woolgar, Eds. (1990) Representation in Scientific Practice, Cambridge, MA, MIT Press.

McCoyd, J. L. M. and T. S. Kerson (2006) 'Conducting intensive interviews using email', Qualitative Social Work, vol. 5, no. 3, pp. 389-406.

Mort, M., C. R. May and T. Williams (2003) 'Remote doctors and absent patients: acting at a distance in telemedicine?’, Science, Technology \& Human Values, vol. 28, no. 2, pp. 274-295. 
Orgad, S. S. (2005) 'From online to offline and back: moving from online to offline relationships with research informants', in Virtual Methods: Issues in Social Research on the Internet, ed. C. Hine, Oxford, Berg, pp. 51-65.

Scoble, M. (2010) ‘Rationale and value of natural history collections digitisation’, Biodiversity Informatics, vol. 7, no. 2, pp. 77-80.

Smith, A. (1999) Why Digitize? Washington D.C., Council on Library and Information Resources. http://www.clir.org/pubs/reports/pub80-smith/reports/pub80smith/pub80.pdf (Retrieved 14 February 2012)

Terras, M. M. (2008) Digital Images for the Information Professional, Aldershot, Ashgate.

The Virtual Knowledge Studio (2008) 'Messy shapes of knowledge - STS explores informatization, new media and academic work', in The Handbook of Science and Technology Studies, ed. E. J. Hackett, O. Amsterdamsaka, M. Lynch and J. Wajcman, Cambridge, MA, MIT Press, pp. 319-351.

Vollmar, A., J. A. Macklin and L. S. Ford (2010) 'Natural history specimen digitization: challenges and concerns', Biodiversity Informatics, vol. 7, no. 2, pp. 93-112. 
Table 1. Participants recruited by appeal to the Taxacom list

\begin{tabular}{|c|c|c|c|c|c|}
\hline $\begin{array}{l}\text { Participant } \\
\#\end{array}$ & Gender $^{i}$ & Country & $\begin{array}{l}\text { User or } \\
\text { resource } \\
\text { developer? }\end{array}$ & $\begin{array}{l}\text { Taxonomic } \\
\text { specialism }\end{array}$ & $\begin{array}{l}\text { Institutional } \\
\text { location }^{\text {ii }}\end{array}$ \\
\hline 1 & $\mathrm{~m}$ & Australia & $\begin{array}{l}\text { Resource } \\
\text { developer }\end{array}$ & $\begin{array}{l}\text { Museum curator } \\
\text { (multiple groups) }\end{array}$ & Museum \\
\hline 2 & $\mathrm{~m}$ & US & \begin{tabular}{l|} 
Resource \\
developer/user
\end{tabular} & Botanist & $\begin{array}{l}\text { University } \\
\text { department }\end{array}$ \\
\hline 3 & $\mathrm{~m}$ & Australia & $\begin{array}{l}\text { Standards } \\
\text { developer }\end{array}$ & Botanist & retired \\
\hline 4 & $\mathrm{f}$ & US & \begin{tabular}{l|} 
Resource \\
developer/user
\end{tabular} & Botanist & $\begin{array}{l}\text { University } \\
\text { herbarium }\end{array}$ \\
\hline 5 & $\mathrm{~m}$ & US & User & Botanist & $\begin{array}{l}\text { University } \\
\text { herbarium }\end{array}$ \\
\hline 6 & $\mathrm{~m}$ & Uruguay & User & Paleontologist & $\begin{array}{l}\text { University } \\
\text { department }\end{array}$ \\
\hline 7 & $\mathrm{~m}$ & US & $\begin{array}{l}\text { Resource } \\
\text { developer/user }\end{array}$ & Nematologist/botanist & $\begin{array}{l}\text { University } \\
\text { department }\end{array}$ \\
\hline 8 & $\mathrm{~m}$ & US & $\begin{array}{l}\text { Resource } \\
\text { developer/user }\end{array}$ & Icthyologist & Museum \\
\hline 9 & $\mathrm{~m}$ & US & $\begin{array}{l}\text { Resource } \\
\text { developer }\end{array}$ & Protistologist & \\
\hline 10 & $\mathrm{~m}$ & US & N/A & Funder & $\begin{array}{l}\text { Funding } \\
\text { body }\end{array}$ \\
\hline
\end{tabular}




\begin{tabular}{|l|l|l|l|l|l|}
\hline 11 & f & US & User & Botanist & $\begin{array}{l}\text { Botanic } \\
\text { garden }\end{array}$ \\
\hline 12 & f & US & User & Botanist & $\begin{array}{l}\text { University } \\
\text { department }\end{array}$ \\
\hline 13 & m & US & $\begin{array}{l}\text { Resource } \\
\text { developer }\end{array}$ & Botanist & University \\
herbarium
\end{tabular}


${ }^{\mathrm{i}}$ Gender was assumed based on standard gendered naming conventions.

ii Taxonomic specialism and institutional location are deliberately non-specific in order to avoid inadvertently identifying participants 